\title{
ANGIOGENESIS IN BOTH NORMAL AND PATHOLOGICAL DEVELOPMENT
}

\author{
BY \\ Mahmoud Galal ${ }^{\mathrm{a}}$, Mohey Elmazar ${ }^{\mathrm{b}}$, Amany AE Ahmed ${ }^{\mathrm{a}}$, Rehab Kamel ${ }^{\mathrm{a}}$, \\ FROM \\ a: Department of Pharmacology and Toxicology, Faculty of Pharmacy, Helwan University, \\ Cairo, Egypt \\ b. Faculty of Pharmacy, The British University in Egypt, Cairo, Egypt
}

\begin{abstract}
Angiogenesis is the formation and development of new blood vessels from preexisting vasculature. Inadequate balance of angiogenesis can cause various pathological disorders and considered mainly in cancer development. The blood vessels in solid tumors have abnormal physiological and morphological characteristics compared to the normal vasculatures. Two main gene expressions (matrix metalloproteinases-9) and (tumor necrosis factor- $\alpha$ ) have very critical role in development of angiogenesis associated with cancer as well as its further metastasis. As stem cells are considered the source for all cell differentiation in every process in the body, they also are the source for the whole process of tumor tissues development. Stem cells participate in angiogenesis by differentiation into vascular lineage and/or by releasing angiogenic factors. Cancer stem cells are tumor initiating cells and predicted as a critical population of tumor cells which might mainly drive each step of tumor development.
\end{abstract}

\section{Angiogenesis:}

The term 'angiogenesis' refers to formation and development of new blood vessels (neovascularization) from the pre-existing vasculature. Angiogenesis is a complex multistep process comprised of endothelial cell proliferation, migration, differentiation and remodelling of the extracellular matrix. Capillaries are formed by endothelial cells creating primitive tubules, which are then further supported and augmented by interactions with vascular pericytes. Angiogenesis consists of different steps including endothelial cell proliferation, stimulation of endothelial cells by various endogenous growth factors, cell migration and capillary tube formation (Yancopoulos et al., 2000).

The multi-step process of angiogenesis plays an important role in various simple and complex physiological process starting from embryonic development till various pathological and physiological processes. Under normal circumstances, angiogenesis is observed during inflammation, tissue regeneration and development, organ growth and repair, wound healing, menstrual cycle and metastasis. (Johnson and Traci, 2014; Birbrair et al., 2015).

Balance of angiogenesis when is changed can cause various disorders including malignancy, ischemic diseases, immune disorders and infectious diseases. The balance 
between pro- and anti-angiogenic signals is the key factor that can maintain the overall balance of angiogenesis (Rajabi and Mousa 2017).

Excessive angiogenesis is commonly associated with many pathological diseases, including diabetic retinopathy (Praidou et al., 2010), vascular malformations, atherosclerosis, obesity, arthritis, psoriasis as well as cancer (Dudek et al., 2012). Insufficient blood supply in case of reduced angiogenesis imposes the risk of tissue death in several diseases such as ischemic chronic wound (Frykberg and Banks, 2015) and coronary artery diseases (Kastrup, 2010). It is also indicated in poor bone integration and survival in fracture repair (Saran et al., 2014).

\section{Cancer and Angiogenesis:}

Angiogenesis plays a vital role in tumor growth and progression, survival and metastasis. A primary tumor can grow only $1-2 \mathrm{~mm}^{3}$; beyond that it needs vascularization or angiogenesis to grow further. Moreover, tumor cells trigger an "angiogenic switch" that attracts blood vessels from the nearby stroma, which is further controlled by several pro- or anti-angiogeneic factors (Caporarello et al., 2017; Mukherjee, 2018). Several lines of evidence have indicated that growth, persistence and metastasis of solid tumors is dependent upon angiogenesis. Furthermore, a lot of studies have indicated that angiogenesis is also involved in the pathogenesis of haematopoietic malignancies. Because of this association of angiogenesis with multiple and diverse tumor types, it has long been a goal of clinicians to disrupt angiogenesis as a novel strategy for cancer therapy (Finney et al., 2009).

The processes by which drugs inhibit tumor growth by suppressing angiogenesis is called antiangiogenesis procedure and is considered one of the most potential cancer treatment strategies. Several studies showed that significant therapeutic advantage can be achieved by treatment with angiogenesis inhibitors such as sorafenib, sunitinib, and bevacizumab (Escudier et al., 2007; Faivre et al., 2007).

Although it was thought for many years that spread of cancer cells and growth of localized tumors beyond a few millimeters in size require local angiogenesis in which tumor cells produce new blood vessels by releasing pro-angiogenic chemical signals, recent studies have reported that tumors of brain, lung, and liver can grow along existing vessels without evoking new vessel growth (Rieger and Welter 2015; Winkler, 2017). Normal cells proximal to cancer cells may also support a pro-angiogenic response via signaling molecules. Local neovascularization supplies growing tumors with oxygen and essential nutrients, supports tumor extension and invasion into nearby normal tissue, and is essential to distant metastasis (Rajabi and Mousa, 2017). The growth of blood vessels is essential for organ growth and repair while the lymphatic vasculature forms the vessel network that participates in immune defense. Both angiogenesis and lymphangiogenesis play an important role in the process of numerous malignances (Alitalo et al., 2005; Carmeliet, 2005).

\section{Tumor Vasculatures Characteristics:}

The solid tumors have blood vessels with abnormal physiological and morphological characteristics compared to the normal vasculatures. Tumor vasculatures 
have highly chaotic and irregular arrangement of blood vessels (Morikawa et al., 2002; Campbell, 2006). Tumors blood vessels have increased permeability to circulating macromolecules and significantl higher growth rate of endothelial cells. The enhanced microvessel permeability is likely associated with creation of abnormally opened interendothelial junctions (Bae et al., 2011) (Fig. 1). These characteristics of tumor vasculatures has led to design and develop a number of effective anticancer drugs. In tumors the size of vascular gap openings falls within the range of 400 to $600 \mathrm{~nm}$ which can be targeted by nano-structured materials of smaller sizes to penetrate into leaky tumor vasculatures delivering the loaded anti-cancer agents. (Bae et al., 2011)

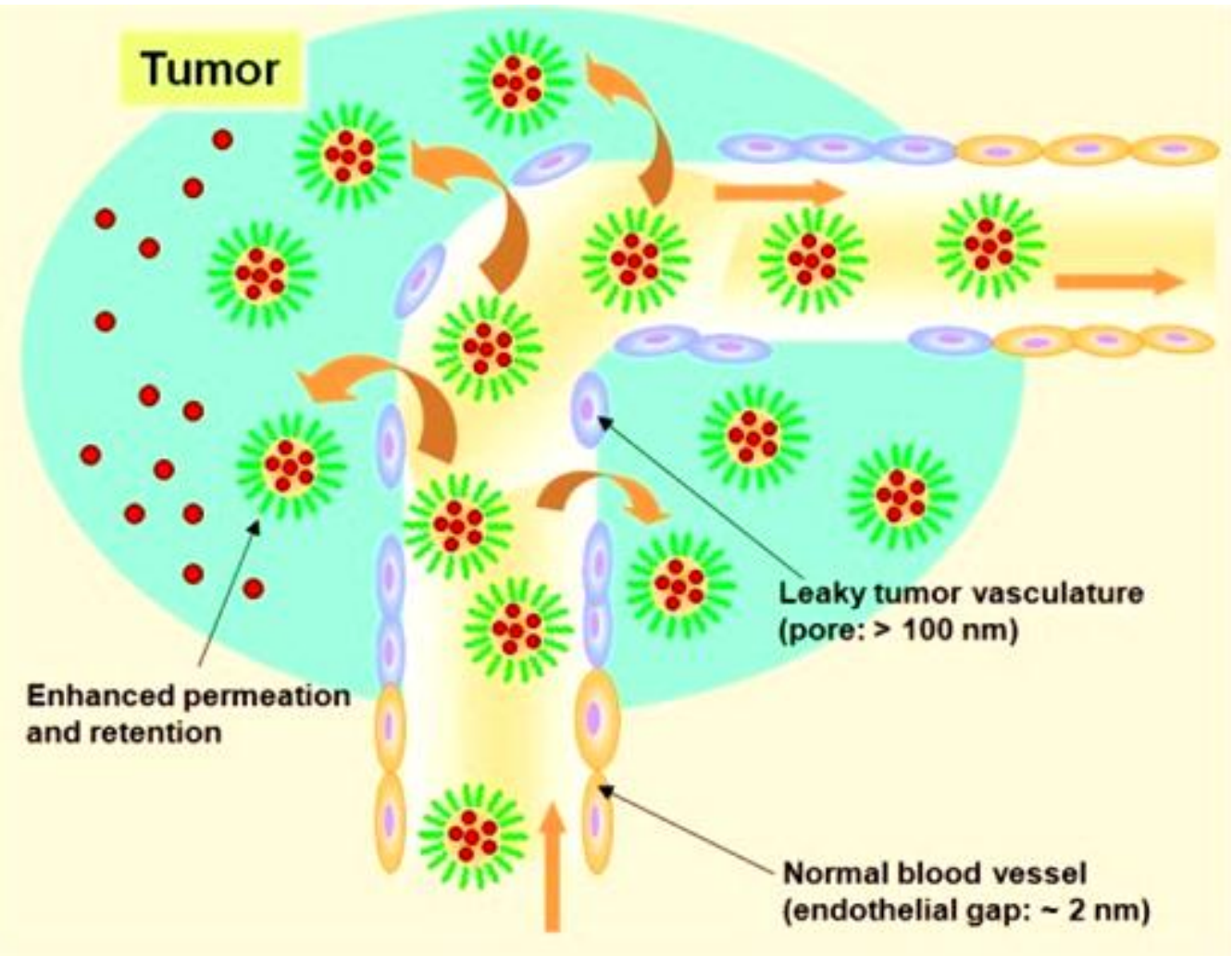

Fig. 1:Schematic illustration depicting the cancer-targeted accumulation of nanovehicles via the enhanced permeability and retention (EPR) effect (Bae et al., 2011).

\section{Matrix Metalloproteinases (MMPs):}

The establishment and progression of metastasis is a complex multi-step process involving dynamic interactions between cancer cells and their microenvironment (Egeblad et al., 2010; Hanahan and Weinberg, 2011; Valastyan and Weinberg, 2011). Within the primary tumor, these reciprocal interactions initiate angiogenic vessel assembly and culminate in the formation of vascular networks contributing to both tumor growth and cancer cell spread to secondary sites (Weis and Cheresh, 2011). This metastasis-supporting vasculature is shaped in part through continuous proteolytic modifications of tissue 
extracellular matrix and various cell surface molecules, particularly by the matrix metalloproteinases (MMPs). Nevertheless, the precise mechanisms whereby MMP-9 and other MMPs induce development and sustain those distinct angiogenic vessels capable of supporting tumor cell intravasation and metastatic dissemination of aggressive cancer cells, are still in the spotlight of cancer research. (Deryugina and Quigley, 2015 )

As an example, MMPs have been implicated in diverse roles in breast cancer development and progression. While many of different MMPs expressed in breast cancer are produced by stromal cells, MMP-9 is produced mainly by the tumor cells themselves (Mehner et al., 2014). The human breast cancer cell-produced MMP-9 is specifically required for invasion in cell culture and for pulmonary metastasis in a mouse orthotopic model of basal-like breast cancer. The tumor cell-produced MMP-9 promotes tumor vascularization with only modest impact on primary tumor growth, and that silencing of MMP-9 expression in tumor cells leads to an altered transcriptional program consistent with reversion to a less malignant phenotype (Mehner et al., 2014). MMP-9 is most highly expressed in human basal-like and triple negative breast cancer and contributes to metastatic progression. MMP-9 may offer a target for anti-metastatic therapies for basallike triple negative breast cancers, a poor prognosis subtype with few available molecularly targeted therapeutic options (Mehner et al., 2014).

\section{Tumor Necrosis Factor- $\alpha$ (TNF- $\alpha)$ :}

Tumor necrosis factor- $\alpha$ (TNF- $\alpha$ ) was originally described as a circulating factor that can induce haemorrhagic necrosis of tumors. It is now clear that TNF- $\alpha$ has many different functions in cancer biology. In addition to causing death of cancer cells, TNF- $\alpha$ can activate cancer cell survival and proliferation pathways, trigger inflammatory cell infiltration of tumors and promote angiogenesis as well as tumor cell migration and invasion (Waters et al., 2013).

Although administration of TNF- $\alpha$ can cause tumor regression in specific rodent tumor models, human expression polymorphisms suggest that TNF- $\alpha$ can be a tumorpromoting cytokine, whereas blocking the TNF- $\alpha$ pathway in a variety of tumor models inhibits tumor growth. In addition to direct effects of TNF- $\alpha$ on tumors, TNF- $\alpha$ can variously affect immunity and tumor microenvironment. Whereas TNF- $\alpha$ can promote immune surveillance designed to eliminate tumors, it can also drive chronic inflammation, autoimmunity, angiogenesis, and other processes that promote tumor initiation, growth and spread (Lebrec et al., 2015). TNF- $\alpha$ signal transduction pathways can open the way for more selective targeting TNF- $\alpha$ signaling in cancer therapy (Waters et al., 2013).

\section{Stem Cells:}

Stem cells are classically defined as cells that have the capacity to differentiate into specialized cell types and the ability to self-renew, by dividing, while maintaining an undifferentiated state. An example of most important stem cells type are embryonic stem cells (ESs) which are considered pluripotent with an intrinsic ability to differentiate into all three germ layers (King et al., 2014). 


\section{Stem Cells and Angiogenesis:}

Stem cells participate in angiogenesis by differentiating into vascular lineage and/or by releasing angiogenic factors. A number of stem and progenitor cells have been examined for boosting angiogenesis within ischemic cardiovascular diseases, including adult bone marrow-derived mononuclear cells, endothelial progenitor cells, and pluripotent stem cell derived endothelial cells. Stem cells have been shown to play an important role both through direct differentiation and incorporation as mature endothelial cells, as well as, a potent source of pro-angiogenic growth factors that support new vessels formation (Hou et al., 2016). There are three main populations of endothelial progenitors derived from adult tissue that demonstrate potential augmenting vasculogenesis in wound healing including endothelial progenitor cells (EPCs), mesenchymal stem cell (MSCs), and inducedpluripotent stem (iPS) cells (Fig. 2) (King et al., 2014). MSCs were reported to have the ability to differentiate into endothelial lineage and/or express endothelial lineage markers (Huang et al, 2010). Also MSCs secrete angiogenic paracrine factors such as vascular endothelial growth factor (VEGF), basic fibroblast growth factor (bFGF), and plateletderived growth factor (PDGF) that stimulate neovessel formation (Gnecchi et al., 2006). These varying mechanisms enable stem cells to differentiate and/or to incorporate into vasculature or release paracrine factors to stimulate host-derived angiogenesis.

\section{Cancer Stem Cells and Angiogenesis:}

Cancer is a complicated disease in which it has two main phenomena; abnormal cells that divide without control and the ability of that cells to invade other tissues, spreading to other parts of the body through the blood and lymph, developing to the most devastating stage (metastasis) and considered the leading cause of death in cancer.

The most systemic anticancer therapies after primary tumor resection often fail because of tumor recurrence and over-occurring side effects. Increasing evidence indicates existence of small subpopulations of cells endowed with unique self-renewal capacity, tumorigenesis, and radio- or chemotherapy resistance, which defined as cancer stem cells (CSCs) or tumor initiating cells. These cells are predicted as a critical population of tumor cells which might mainly drive each step of tumor programming, including neoplasm initiation, tumor growth and metastasis (Lapidot et al., 1994; Al-Hajj et al., 2003; Singh et al., 2004). Initially, cancer stem cells were identified in hematological malignancies and then they were isolated subsequently from many tumors including breast, prostate, brain, gastrointestinal, liver, lung, skin and ovarian cancer. 


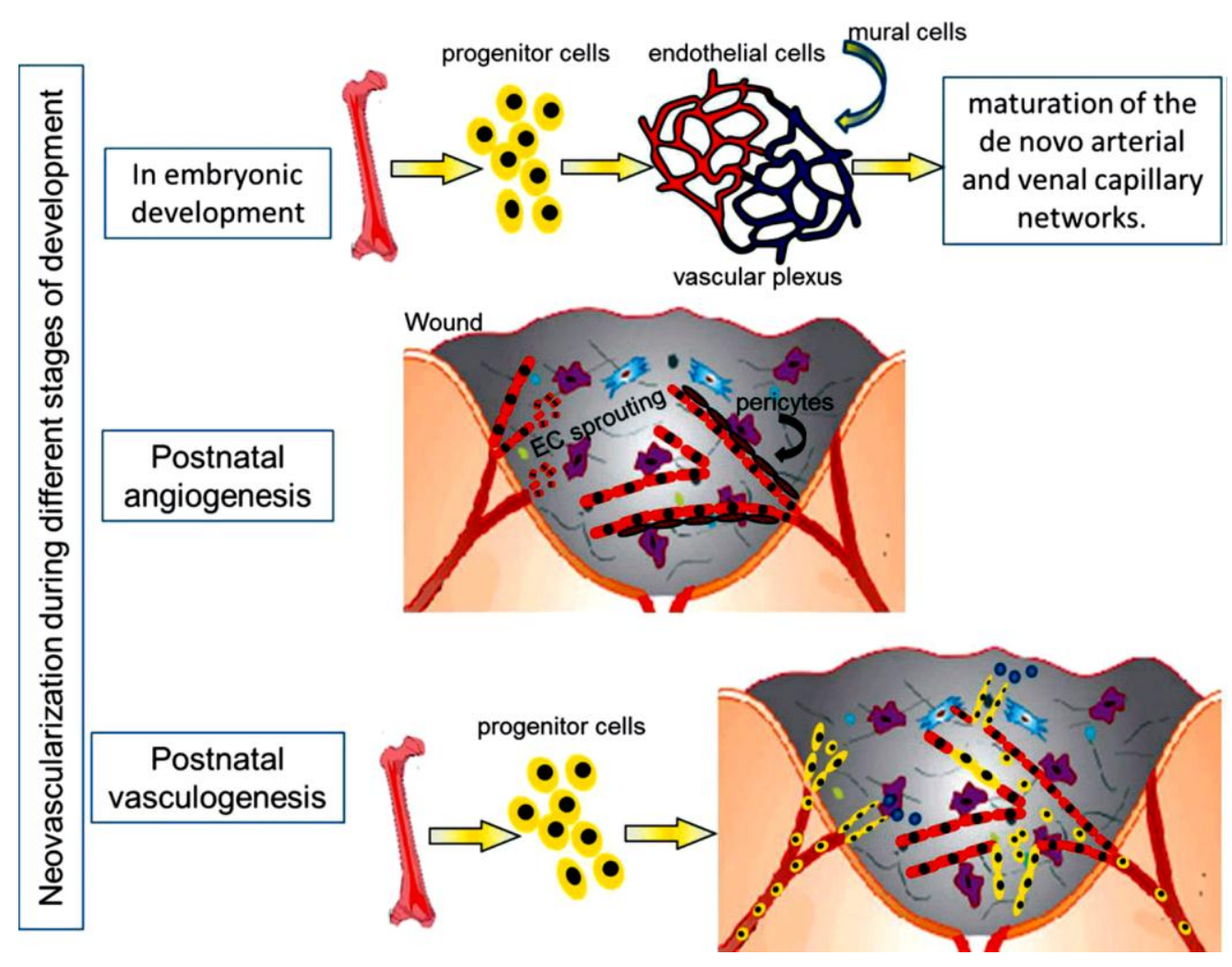

Fig. 2:Neovascularization during different stages of development. In embryonic development, the primary precursor cells are termed angioblasts and hemangioblasts that migrate, differentiate to endothelial cells, and then coalesce to form the vascular plexus and de novo vasculature. In the postnatal setting, the formation of new blood vessels occurs primarily through two independent processes: angiogenesis and vasculogenesis. Angiogenesis results from the sprouting of new capillaries from existing vessels. This occurs through endothelial cell (EC) activation, proliferation, and migration to form new vessels that are then stabilized by pericytes. Vasculogenesis describes de novo generation of vessels, a process dependent on bone-marrow-derived and circulating endothelial progenitor cells (EPCs), which differentiate to endothelial cells and result in formation of new vessels. The differentiated endothelial cells also participate in reendothelialization and vessel repair (King et al., 2014).

Accumulating evidence has shown that CSCs interact closely with angiogenesis, especially in glioma, a solid tumor that is highly lethal, difficult to treat and intimately dependent on angiogenesis. A lot of studies revealed the interplay among CSCs, angiogenesis and tumor vasculature based on this tumor model. (Zhao et al., 2011).

Tumor angiogenesis is critical for tumor growth and maintenance (Kaur et al., 2005), that is important cellular parameter of tumorigenesis. CSCs show greater potential for tumor initiation and formation than non-stem cell like tumor cells. Gliomas are examplary 
for the significant impact of angiogenesis on tumor progression with impressively elevated levels of VEGFs. Although VEGFs are expressed at elevated levels by endothelial cells engaged in angiogenesis and circulating bone marrow derived endothelial progenitor cells, many types of human cancer cells can also secrete VEGFs (Kerbel, 2008). In these regards, it is logical to suggest that cancer stem cells can function on angiogenesis. An emerging area of research supports that CSCs, besides their self-renewal and proliferative capabilities, may promote tumor angiogenesis (Zhao et al., 2011).

\section{Chorioallantoic Membrane (CAM) as an Experimental Model for Assessment of Angiogenesis:}

The main challenge for studying angiogenesis was to find a suitable model for its assessment. Hen's Egg Test Chorioallantoic Membrane (HET-CAM) is considered the most suitable model for angiogenic related assays. CAM is an extraembryonic membrane which serves as a gas exchange surface and its function is supported by a dense capillary network (Fig. 3). It is a highly vascularized membrane that surrounds the chick embryo (Ribatti, 2012). CAM assay is considered an experimental alternative to animal model. It serves as an ideal model for studying pro- or anti-angiogenic properties of different compounds. CAM provides an effective model for grafting cell lines, tissues as well as tumors. Tumor grafting on CAM gives the opportunity for studying several parameters for tumor growth such as mass formation, induced angiogenesis and metastasis independently or combined with anti-cancer compounds. Among the most valuable features of CAM assay are inexpensive, relative ease of carrying out the assay, high sensitivity for very low quantities of tested compound and allowing live real time monitoring. HET-CAM model was subjected to several trials making it the selected model for both quantitative and qualitative assessments for angiogenesis and tumor cultivation (Galal et al., 2016).

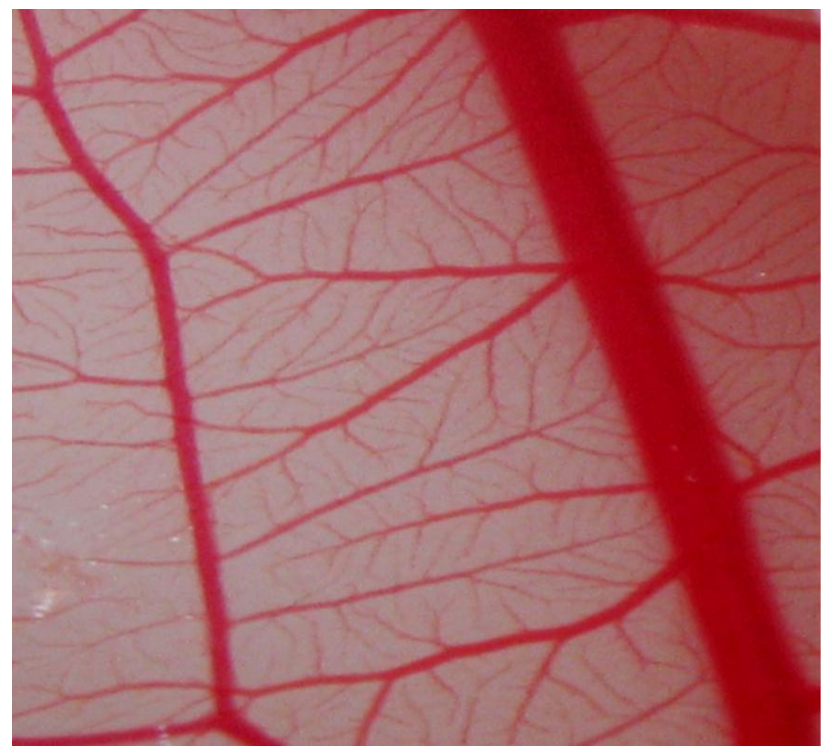

Fig. 3:Live image for Chorioallantoic Membrane (CAM) blood vessels network (Galal et al., 2016). 


\section{REFERENCES:}

Al-Hajj M, Wicha MS, Benito-Hernandez A, Morrison SJ, Clarke MF (2003). Prospective identification of tumorigenic breast cancer cells. Proc Natl Acad Sci USA; 100:3983-3988.

Alitalo K, Tammela T, Petrova TV (2005). Lymphangiogenesis in development and human disease. Nature; 438:946-953.

Bae K, Chung H, Park T (2011). Nanomaterials for cancer therapy and imaging. Mol. Cells; 31:295-302.

Birbrair A, Zhang T, Wang ZM, Messi ML, Mintz A, Delbono O (2015). Pericytes at the intersection between tissue regeneration and pathology. Clin. Sci.; 128(2):81-93.

Campbell RB (2006). Tumor physiology and delivery of nanopharmaceuticals. Anticancer Agents Med. Chem.; 6, 503-512.

Caporarello N, Lupo G, Olivieri M, Cristaldi M, Cambria MT, Salmeri M, Anfuso CD (2017). Classical VEGF, Notch and Ang signalling in cancer angiogenesis, alternative approaches and future directions (Review). Mol Med Rep; 16(4), 43934402 .

Carmeliet P (2005). Angiogenesis in life, disease and medicine. Nature; 438:932-936.

Deryugina EI, Quigley JP (2015). Tumor Angiogenesis: MMP-Mediated Induction of Intravasation- and Metastasis-Sustaining Neovasculature. Matrix Biol.; 44-46:94112.

Dudek AZ, Gupta K, Ramakrishnan S, Mukhopadhyay D (2012). Tumor Angiogenesis 2012. J. Oncol.; 2012:857383.

Egeblad M, Nakasone ES, Werb Z (2010). Tumors as organs: complex tissues that interface with the entire organism. Dev Cell.; 18:884-901.

Escudier B, Eisen T, Stadler WM, Szczylik C, Oudard S, Siebels M, Negrier S, Chevreau C, Solska E, Desai AA, Rolland F, Demkow T, Hutson TE, Gore M, Freeman S, Schwartz B, Shan M, Simantov R, Bukowski RM; TARGET Study Group (2007). Sorafenib in advanced clear-cell renal-cell carcinoma. N Engl J Med; 356:125-134.

Faivre S, Demetri G, Sargent W, Raymond E (2007). Molecular basis for sunitinib efficacy and future clinical development. Nat Rev Drug Discov; 6:734-745.

Finney L, Vogt S, Fukai T, Glesne D (2009). Copper and angiogenesis: unravelling a relationship key to cancer progression. Clin Exp Pharmacol Physiol; 36(1):88-94.

Frykberg R, Banks J (2015). Challenges in the Treatment of Chronic Wounds. Adv. Wound Care (New Rochelle); 4(9):560-582.

Galal M, El-Khatib A, Elmazar M (2016). Assessment of Hen's Egg Test Chorioallantoic Membrane (HET-CAM) for screening of anticancer activities of drugs. ALTEX Alternatives to Animal Experimentation; 5(1):156. 
Gnecchi M, He H, Noiseux N, Liang OD, Zhang L, Morello F, Mu H, Melo LG, Pratt RE, Ingwall JS, Dzau VJ (2006). Evidence supporting paracrine hypothesis for Akt-modified mesenchymal stem cell-mediated cardiac protection and functional improvement. FASEB J; 20: 661-669.

Hanahan D, Weinberg RA (2011). Hallmarks of cancer: the next generation. Cell.; 144:646-674.

Hou L, Kim JJ, Woo YJ, Huang NF (2016). Stem cell-based therapies to promote angiogenesis in ischemic cardiovascular disease. Am J Physiol Heart Circ Physiol.; 310:H455-H465.

Huang NF, Chu J, Lee RJ, Li S (2010). Biophysical and chemical effects of fibrin on mesenchymal stromal cell gene expression. Acta Biomater; 6:3947-3956.

Johnson KE, Traci A (2014). WilgusVascular Endothelial Growth Factor and Angiogenesis in the Regulation of Cutaneous Wound Repair. Adv. Wound Care. (New Rochelle); 3(10): 647-661.

Kastrup J, (2010). Gene therapy and angiogenesis in patients with coronary artery disease. Expert Rev. Cardiovasc. Ther.; 8(8): 1127-1138.

Kaur B, Khwaja, FW, Severson EA, Matheny SL, Brat DJ, Van Meir EG (2005). Hypoxia and the hypoxia-inducible-factor pathway in glioma growth and angiogenesis. Neuro-Oncology; 7: 134-153.

Kerbel RS (2008). Molecular origins of cancer: Tumor angiogenesis. N Engl J Med; 358: 2039-2049.

King A, Balaji S, Keswani SG, Crombleholme TM (2014). The Role of Stem Cells in Wound Angiogenesis. Adv Wound Care (New Rochelle); 3(10):614-625.

Lapidot T, Sirard C, Vormoor J, Murdoch B, Hoang T, Caceres-Cortes J, Minden M, Paterson B, Caligiuri MA, Dick JE (1994). A cell initiating human acute myeloid leukaemia after transplantation into SCID mice. Nature; 367: 645-648.

Lebrec H, Ponce R, Preston BD, Iles J, Born TL, Hooper M (2015). Tumor necrosis factor, tumor necrosis factor inhibition, and cancer risk. Curr Med Res Opin.; 31:557-574.

Mehner C, Hockla A, Miller E, Ran S, Radisky DC, Radisky ES (2014). Tumor cellproduced matrix metalloproteinase 9 (MMP-9) drives malignant progression and metastasis of basal-like triple negative breast cancer. Oncotarget.; 5(9):2736-2749.

Morikawa S, Baluk P, Kaidoh T, Haskell A, Jain RK, McDonald DM (2002). Abnormalities in pericytes on blood vessels and endothelial sprouts in tumors. Am. J. Pathol.; 160, 985-1000.

Mukherjee S (2018). Recent progress toward antiangiogenesis application of nanomedicine in cancer therapy. Future Sci OA.;4(7):FSO318. 
Praidou A, Androudi S, Brazitikos P, Karakiulakis G, Papakonstantinou E, Dimitrakos S (2010). Angiogenic growth factors and their inhibitors in diabetic retinopathy. Curr. Diabetes Rev.; 6(5): 304-312.

Rajabi M, Mousa SA (2017). The Role of Angiogenesis in Cancer Treatment. Biomedicines; 5(2):34.

Ribatti D (2012). Chicken chorioallantoic membrane angiogenesis model. Methods Mol Biol.; 843:47-57.

Rieger H, Welter M (2015). Integrative models of vascular remodeling during tumor growth. Wiley Interdiscip Rev Syst Biol Med.;7(3):113-129.

Saran U, Gemini Piperni S, Chatterjee S (2014). Role of angiogenesis in bone repair. Arch. Biochem. Biophys.; 561:109-117.

Singh SK, Hawkins C, Clarke ID, Squire JA, Bayani J, Hide T, Henkelman RM, Cusimano MD, Dirks PB (2004). Identification of human brain tumour initiating cells. Nature; 432: 396-401.

Valastyan S, Weinberg RA (2011). Tumor metastasis: molecular insights and evolving paradigms. Cell.; 147:275-292.

Waters JP, Pober JS, Bradley JR (2013). Tumour necrosis factor and cancer. J Pathol.; 230:241-248.

Weis SM, Cheresh DA (2011). Tumor angiogenesis: molecular pathways and therapeutic targets. Nat Med.; 17:1359-1370.

Winkler F (2017). Hostile takeover: How tumors hijack pre-existing vascular environments to thrive. J. Pathol.; 242(3):267-272.

Yancopoulos GD, Davis S, Gale NW, Rudge JS, Wiegand SJ, Holash J (2000). Vascular-specific growth factors and blood vessel formation. Nature; 407(6801):242-248.

Zhao Y, Bao Q, Renner A, Camaj P, Eichhorn M, Ischenko I, Angele M, Kleespies A, Jauch KW, Bruns C (2011). Cancer stem cells and angiogenesis. Int. J. Dev. Biol.; $55: 477-482$. 


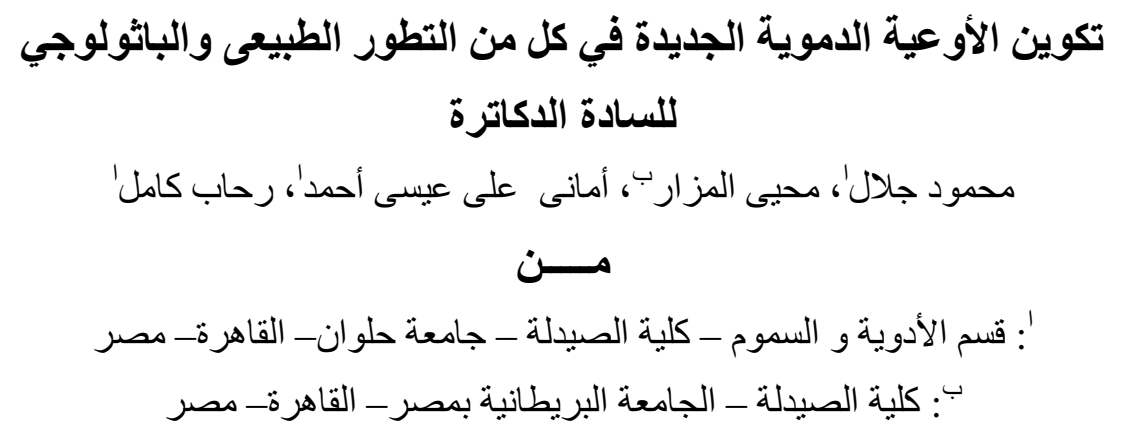

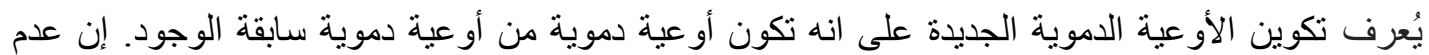

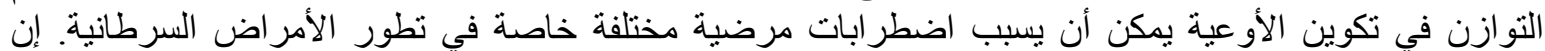

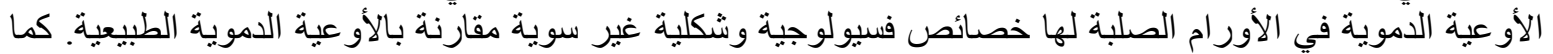

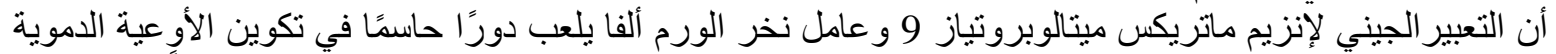

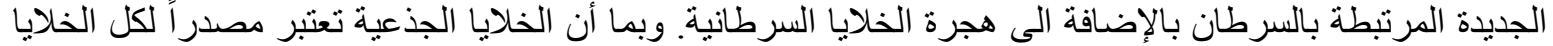

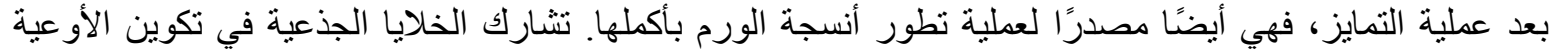

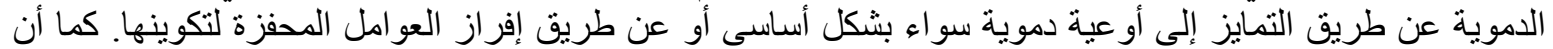

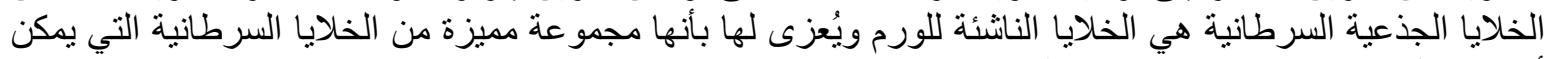
أن نقود كل خطوة من خطو ات تطور الورم. 